Results There were 28 instances of buried FREKA PEGs among 21 patients. Nowadays, our usual management is to deploy a new PEG in a separate site, then attempt endoscopic bumper removal using a wire-guided $15 \mathrm{Fr}$ dilatation balloon introduced via the cut stump of the buried PEG (balloon push technique). The balloon dilates the track and grips the internal surface of the bumper during forward traction.

Conscious sedation was used in 21 instances and general anaesthesia in 7. It was possible to deploy a new PEG in 26 instances (93\%) and to unbury the bumper endoscopically in 24 instances (86\%). An external (push-pull) $\mathrm{T}$ snare technique was required twice.

There were no immediate complications; all but one survived for at least 1 year and 11 patients (52\%) remain alive after 1-130 months. Three required surgical removal \pm deployment of a new PEG. A patient for whom the bumper stump was left alone and a new PEG deployed had recurrent infections at the stump site during his three remaining years.

Conclusions Buried PEG bumpers can normally be managed safely endoscopically. If this fails, and the patient is expected to survive, surgery should be done to remove them. General anaesthesia was needed in around a quarter. Long term survival may be expected in some patients. Although buried bumpers could be avoided with meticulous daily care of these patients, when encountered appropriate intervention is necessary to deal with this uncommon complication associated with PEG tube feeding.

\section{PTH-038 RESONANCE ENHANCED SELF-PROPELLED CAPSULE ENDOSCOPY FOR SMALL BOWEL EXAMINATION}

${ }^{1}$ Yang Liu*, 'Bingyong Guo, ${ }^{2}$ Shyam Prasad. ${ }^{1}$ University of Exeter, Exeter, UK; ${ }^{2}$ The Royal Devon and Exeter NHS Foundation Trust, Exeter, UK

\subsection{6/gutjnl-2019-BSGAbstracts.63}

Introduction Capsule endoscopy has become established as the primary modality for examining the small intestinal mucosa. However, its reliance on peristalsis for passage through the intestine causes variable locomotion speeds, which can lead to incomplete visualisation of the mucosa and potentially missed pathology. In addition, lengthy videos resulting from long transit times can be both time-consuming and burdensome for clinicians to examine.

Methods A resonance enhanced self-propelled capsule endoscope (Liu et al., Int. J. Mech. Sci., 66:2-11, 2013; Liu et al., Nonlinear Dyn., 83:1029-1041, 2016) was developed for small bowel examination. The driving principle of this technique is that rectilinear motion of the capsule can be generated using a periodically driven internal mass interacting with the capsule's main body (as a hammer), in the presence of intestinal resistances. A small 'hammer' actuator, which was excited by an on-off square wave signal, was mounted in the capsule prototype. The capsule can perform forward and backward progression by modulating the amplitude and frequency of the square wave excitation. Early proof-of-concept tests in a laboratory environment were carried out for different capsuleintestine contact conditions by utilising a synthetic small intestine.

Results Three contact scenarios were investigated: capsule moving on an aluminium bench (Case 1), on a flat synthetic small intestine (Case 2), and in a tubular synthetic small intestine (Case 3). Extensive tests under different control parameters (e.g. varying the frequency of the excitation between 1-20 Hz), were conducted for each case, and the main results are: (1) The maximum average speed of forward progression was $5 \mathrm{~mm} / \mathrm{s}$ for Case $1,7 \mathrm{~mm} / \mathrm{s}$ for Case 2, and $2 \mathrm{~mm} / \mathrm{s}$ for Case 3. (2) In all three cases, the maximum average speed of backward progression was $1 \mathrm{~mm} / \mathrm{s}$. (3) The energy efficiency of the capsule was up to $1.5 \mathrm{~mm} / \mathrm{J}$.

Conclusions Assuming a maximum small intestinal length of 6 $\mathrm{m}$ and capsule speed of just $2 \mathrm{~mm} / \mathrm{s}$ (Case 3), small intestinal transit could be reduced to no more than 50 minutes, offering the potential for a 'live' and controllable examination. The energy efficiency of the prototype equates to a total of $4 \mathrm{~kJ}$ energy for a complete small bowel examination, which could be provided by currently available button cells.

\section{PTH-039 THE COST-EFFECTIVENESS OF RADIOFREQUENCY ABLATION FOR GASTRIC ANTRAL VASCULAR ECTASIA REFRACTORY TO FIRST-LINE ENDOSCOPIC THERAPY}

\begin{abstract}
${ }^{1,2}$ Cormac Magee*, ${ }^{1,2}$ David Graham, ${ }^{4}$ Jessica McMaster, ${ }^{3}$ Catherine Leonard, ${ }^{4}$ Heather Davies, ${ }^{1}$ Matthew Banks, ${ }^{1,2}$ Rami Sweis, ${ }^{2}$ Gideon Lipman, ${ }^{2}$ Durayd Alzoubaidi, 1,2Mohamed Hussein, 2Martin Everson, 1,2 Laurence Lovat, 1,2Sarmed Sami, ${ }^{1,2}$ Charles Murray, ${ }^{5}$ Charles Gordon, ${ }^{4}$ Stuart Mealing, ${ }^{6}$ Howard Smart, ${ }^{1,2}$ Rehan Haidry. ${ }^{1}$ University College London Hospitals, London, UK; ' ${ }^{2}$ University College London, London, UK; ${ }^{3}$ Medtronic UK; ${ }^{4}$ York Health Economics Consortium, York, UK; ${ }^{5}$ Royal Bournemouth and Christchurch Hospitals, Bournemouth, UK; ${ }^{6}$ Royal Liverpool University Hospital, Liverpool, UK
\end{abstract}

\subsection{6/gutjnl-2019-BSGAbstracts.64}

Introduction Argon plasma coagulation (APC) is the most commonly used treatment within the UK NHS for Gastric Antral Vascular Ectasia (GAVE), a chronic condition that can cause debilitating symptoms secondary to chronic blood loss. This economic evaluation provides a preliminary assessment of the cost-effectiveness of radiofrequency ablation (RFA) in this patient population, which recent evidence suggests may offer improved safety and effectiveness when used to treat APCrefractory GAVE patients.

Methods A Markov model was constructed to undertake a cost-utility analysis for adults diagnosed with GAVE symptomatic of iron-deficiency anaemia. The economic evaluation used a UK NHS and personal social services (PSS) perspective, with a 20-year time horizon, and three-month cycles. Patients transfer between health states defined by haemoglobin level: mild (11 to $12 \mathrm{~g} \mathrm{dl}^{-1}$ ), moderate ( 9 to $10 \mathrm{~g} \mathrm{dl}^{-1}$ ) and severe $\left(<8 \mathrm{~g} \mathrm{dl}^{-1}\right)$. The clinical effectiveness data were sourced from literature and expert opinion. Resource use and costs were reflective of the UK NHS, and parameter uncertainty was explored using probabilistic sensitivity analysis (PSA). Benefits were qualified using Quality Adjusted Life Years (QALYs), with utility weights taken from the literature. The primary output was the Incremental Cost-Effectiveness Ratio (ICER) expressed as cost per QALY gained.

Results The base case ICER was $£ 13,933$ per QALY gained, with a $58.9 \%$ chance that RFA was cost-effective at a threshold of $£ 20,000$ per QALY gained. The model estimated that implementing RFA would result in reductions in the need for intravenous iron, endoscopies and blood transfusions by $27.1 \%, 32.3 \%$ and $36.5 \%$ respectively. Compared to APC, RFA was associated with an estimated $36.7 \%$ fewer procedures as well as less time in severe health states (1.77 years) and more time in mild health states (1.2 years) when treated with RFA over a 20-year time horizon. 